\title{
Evaluation of Post-Vaccination Symptoms of Two Common COVID-19 Vaccines Used in Abha, Aseer Region, Kingdom of Saudi Arabia
}

\author{
Mohamed Adam (1D' \\ Moawia Gameraddin (D) ${ }^{2}$ \\ Magbool Alelyani (D) \\ Mohammad Y Alshahrani ${ }^{3}$ \\ Awadia Gareeballah (D) ${ }^{2}$ \\ Irshad Ahmad (iD ${ }^{4}$ \\ Abdulrahman Azzawi ${ }^{5}$ \\ Basem Komit $\mathbb{D}^{6}$ \\ Alamin Musa (iD) \\ 'Department of Radiological Sciences, \\ College of Applied Medical Sciences, King \\ Khalid University, Abha, Saudi Arabia; \\ ${ }^{2}$ Department of Diagnostic Radiologic \\ Technology, Faculty of Applied Medical \\ Sciences, Taibah University, Al-Madinah, \\ Saudi Arabia; ${ }^{3}$ Department of Clinical \\ Laboratory Sciences, College of Applied \\ Medical Sciences, King Khalid University, \\ Research Center for Advanced Materials \\ Science (RCAMS), King Khalid University, \\ Abha, Saudi Arabia; ${ }^{4}$ Department of \\ Medical Rehabilitation Sciences, Faculty \\ of Applied Medical Sciences, King Khalid \\ University, Abha, Aseer, Saudi Arabia; \\ ${ }^{5}$ Medical City, King Khalid University, \\ Abha, Aseer, Saudi Arabia; ${ }^{6}$ Medical \\ Imaging Department, Aseer Central \\ Hospital, Ministry of Health, Abha, Saudi \\ Arabia
}

Correspondence: Mohamed Adam Department of Radiological Sciences, College of Applied Medical Sciences, King Khalid University, Abha, Aseer, Saudi Arabia

Email o_maurie2006@yahoo.com
Background: The Pfizer-BioNTech (BNT162b2) and the Oxford-AstraZeneca (ChAdOx1 nCoV-19) COVID-19 vaccines have shown promising safety and acceptability. However, COVID-19 vaccine side effects play an essential role in public vaccine confidence. We aimed to study the side effects of these COVID-19 vaccines.

Methods: A randomized, cross-sectional descriptive study was conducted between March and May of 2021. In total, 330 participants among the King Khalid University community in the Aseer region of the Kingdom of Saudi Arabia reported their side effects following the COVID-19 vaccine. A questionnaire was designed and validated to collect the participants' demographic data and COVID-19-related symptoms after COVID-19 vaccine injection.

Results: Symptoms associated with COVID-19 were reported by 226 participants $(68.5 \%)$. The most common side effects reported by the participants were fever $(n=136,41.2 \%)$, fatigue $(\mathrm{n}=119,36.1 \%)$, headache $(\mathrm{n}=86,24.2 \%)$, malaise $(\mathrm{n}=121,36.7 \%)$, myalgia $(\mathrm{n}=$ $121,36.7 \%)$, and muscle and joint pain $(\mathrm{n}=76,23 \%)$. Of the participants, $5.1 \%$ became infected with COVID-19 after vaccination. Symptoms were significantly more common in males than in females $(\mathrm{p}=0.006)$.

Conclusion: The incidence of COVID-19 vaccination side effects in the Aseer region, Kingdom of Saudi Arabia, was consistent with the manufacturers' data. The most common post-vaccination symptoms reported by the participants were fever, myalgia, malaise, fatigue, muscle and joint pain, and headache. The results of this study showed significant variation in adverse events between Pfizer-BioNTech and Oxford-AstraZeneca COVID-19 vaccines. Healthcare providers and recipients of vaccines can be more confident about the safety of Pfizer-BioNTech and Oxford-AstraZeneca COVID-19 vaccines.

Keywords: COVID-19, vaccines, side effects, symptoms, Pfizer-BioNTech, OxfordAstraZeneca

\section{Introduction}

The global influence of the coronavirus disease 2019 (COVID-19) pandemic has resulted in increased public interest in vaccines. Mass and social media have spread awareness about vaccine importance. However, the incidence of adverse events (side effects) has caused some people to have increased concerns about getting vaccinated, delay getting vaccinated, or strongly oppose vaccination. Due to the ease and speed of the spread of COVID-19 and its infection of the majority of the world's population, awareness of the importance of vaccination has increased. ${ }^{1}$ Various COVID-19 vaccines have exhibited effectiveness to $95 \%$ in counteracting 
symptomatic infections due to coronavirus COVID-19 pandemic. $^{2}$ As of April 2021, 16 vaccines are authorized for public utilization by at least one or more national regulatory authorities. ${ }^{3}$ Saudi Arabia has commenced an early vaccination campaign as a continuation of its outstanding efforts and actions to control the spread of the SARS-CoV-2 virus that causes COVID-19. ${ }^{3,4}$ The ultimate aim of the ministry of health in Saudi Arabia is to vaccinate $70 \%$ of the population. ${ }^{1,5}$

Previously, various studies have reported mild to moderate side effects and reactions to COVID-19 vaccines. ${ }^{6-9}$ These can include mild adverse effects after the first or second doses, including redness or swelling at the injection site, pain, fatigue, fever, muscle pain, nausea, headache, vomiting, chills, and joint pain. ${ }^{8,9}$ Some side effects have been reported for both the Pfizer-BioNTech and Oxford-AstraZeneca vaccines; however, the occurrence of adverse effects is reported to be lower for the Pfizer/BioNTech vaccine as compared to OxfordAstraZeneca and others. ${ }^{10,11}$

This study aimed to evaluate participants' short-term symptoms after receiving either the Pfizer-BioNTech mRNA (BNT162b2) or the Oxford-AstraZeneca (ChAdOx1 nCoV-19) COVID-19 vaccine in the Kingdom of Saudi Arabia. This study will play an important role in vaccine safety by reporting and recognizing post-vaccination symptoms. It will also be beneficial for clinicians and healthcare professionals who must be aware of potential long-term complications and enrich their knowledge about the presence of these possible complications.

\section{Materials and Methods}

\section{Study Design and Sampling}

This cross-sectional study, based on an online survey, was carried out at King Khalid University, Abha, Aseer region of the Kingdom of Saudi Arabia, from March to May of 2021. A questionnaire was designed with the help of an extensive literature review that elucidated the most common questions for collection of this type of data this type of study. ${ }^{12-14}$ After ethical approval from the Deanship of Research, we gave the questionnaire link to the Deanship of E-Learning. A link was displayed on the website for participants to prove that they were associated with King Khalid University. The study population consisted of participants who, during the study period, received either one or two doses of the Oxford-AstraZeneca or Pfizer-
BioNTech vaccine and were at least 18 years of age. Participants who did not receive any vaccination or were not vaccinated with AstraZeneca or Pfizer-BioNTech were excluded from the study.

The study's sample size was calculated using rasoft. com. As the Ministry of Health of Saudi Arabia aims to vaccinate $70 \%$ of the population (approximately 28,000 individuals), a sample size of 330 participants was sufficient to achieve $95 \%$ confidence with a $5.35 \%$ margin of error. A pilot study was performed prior to initiation of this study to ensure the reliability and validity of the questionnaire.

All participants received COVID-19 vaccines during the time of the study. Data was collected using an online survey to evaluate the side effects associated with the vaccines. The survey was distributed to the participants through social media (eg, WhatsApp groups) and e-mails. Three hundred and thirty participants completed the survey (a 33\% response rate).

\section{Data Analysis and Measurement Tool}

The questionnaire was designed following the COVID-19 guidelines of the Ministry of Health of the Kingdom of Saudi Arabia and the World Health Organization, ${ }^{15}$ and it was available in two languages (English and Arabic). It collected information on participants' socio-demographic characteristics, including gender and age, and clinical medical history (eg, diabetes mellitus and hypertension) prior to COVID-19. The other part of the questionnaire focused on COVID-19-vaccine-related data, such as the type of COVID-19 vaccine, the number of doses received, and the onset of side effects within certain time periods.

The dependent variable was the frequency of symptoms (eg, fever, muscle and joint pain, redness at the injection site, myalgia, malaise, fatigue) after injection of COVID-19 vaccines. The participants' onset of symptoms associated with the first and second vaccine doses were determined and compared. The incidence of COVID-19 after vaccination was determined from the Saudi Ministry of Health website. ${ }^{17}$ Thus, frequency and onset of side effects for two common COVID-19 vaccines were analyzed and compared.

\section{Analysis Methods}

Statistical analyses were performed using the Statistical Package for the Social Sciences (SPSS) version 23 (IBM Corp., Armonk, NY, USA). Descriptive statistics were used for qualitative data. A chi-square test $\left(\chi^{2}\right)$ was used to 
compare symptoms between males and females to assess the side effects between the two types of COVID-19 vaccines. Cronbach's $\alpha$ (used to assess the reliability of the questionnaire) was estimated at 0.79 , indicating good reliability. P-values less than 0.05 were considered significant.

\section{Ethical Approval}

This study was approved by the Research Ethics Committee of the College of Medicine, King Khalid University given the number ECM\#2021-4805. Electronic informed consent was obtained from each participant prior to completion of the questionnaire, as requested by the Research Ethics Committee at King Khalid University. All procedures performed in this study involving human participants were performed following the ethical standards of the institutional and national research committees and the Declaration of Helsinki.

\section{Results}

In total, this study included 330 participants (216 [65.5\%] males and 114 [34.5\%] females). The majority of participants $(\mathrm{n}=106,32.1 \%)$ were in the age group of $21-30$ years; $77(23.3 \%)$ were between 31 and 40 years; and 94 $(28.5 \%)$ were between 41 and 50 years (Table 1$)$. Table 1 also summarizes the clinical history of the participants; the majority $(83 \%)$ declared no clinical history of any chronic diseases. Eighteen participants (5.5\%) reported having hypertension, $8(2.4 \%)$ reported having diabetes mellitus, and $8(2.4 \%)$ reported having prior SARS-CoV-2 infection. Other diseases (hypothyroidism, cardiovascular diseases, anaphylaxis asthma, and depression) were less frequent (below 1\%).

Table 2 summarizes the reported side effects of first and second doses of AstraZeneca and Pfizer/BioNTech vaccines. The majority of participants $(n=226,68.5 \%)$ reported no post-vaccination side effects of both doses (Figure 1). The most frequently reported symptoms were fever $(\mathrm{n}=136,41.2 \%)$, fatigue $(\mathrm{n}=119,36.1 \%)$, headache $(\mathrm{n}=86,24.2 \%)$, and muscle and joint pain $(\mathrm{n}=76,23 \%)$, with very few participants reporting redness at the injection site $(\mathrm{n}=4,1.2 \%)$ or seizures $(\mathrm{n}=3,0.9 \%)$ (Table 2$)$.

Concerning the onset of symptoms following the first vs second doses of COVID-19 vaccines, most participants $(n=126,32.1 \%)$ reported no symptoms after the first dose. Most of the symptoms appeared in less than 24 hours after injection $(\mathrm{n}=85,25.8 \%)$ or in the first 15 minutes after injection ( $\mathrm{n}=83,24.2 \%)$. The onset of symptoms differed significantly between the first and second vaccine doses $(\mathrm{p}<$ 0.001) (Table 3). Among the participants, 5.15\% reported
Table I Demographic Characteristics of the Participants and Clinical History

\begin{tabular}{|l|l|l|}
\hline Variables & Frequency & Percent \\
\hline Gender & & \\
Males & 216 & 65.5 \\
Females & 114 & 34.5 \\
\hline Age groups & & \\
I8-20 years & 16 & 4.8 \\
2I-30 years & 106 & 32.1 \\
3I-40 years & 77 & 23.3 \\
4I-50 years & 94 & 28.5 \\
> 50 years & 37 & 11.2 \\
Total & 330 & 100.0 \\
\hline Clinical history & & \\
No clinical history & 274 & 83.0 \\
Diabetes mellitus & 8 & 2.4 \\
Hypertension & 18 & 5.5 \\
Diabetes and hypertension & 8 & 2.4 \\
Prior SARS-COVID-I9 & 8 & 2.4 \\
Immune disease & 2 & 0.6 \\
Hypothyroidism & 1 & 0.3 \\
Hypertension and CVD & 1 & 0.3 \\
Diabetes mellitus and immune disease & 2 & 0.6 \\
Cardiovascular diseases & 1 & 0.3 \\
History of anaphylaxis & 2 & 0.6 \\
Thyroidectomy & 1 & 0.3 \\
CVD with diabetes mellitus and & 1 & 0.3 \\
hypertension & 2 & \\
Asthma & 1 & 0.6 \\
Depression & 0.3 \\
Total & 17.0 \\
\hline
\end{tabular}

getting COVID-19 after vaccination (Figure 2). The postvaccination symptoms were significantly higher in participants who received the Oxford-AstraZeneca vaccine than in those who received the Pfizer-BioNTech vaccine $(\mathrm{p}=0.022)$ (Table 4). Notably, symptoms were significantly higher in males than in females $(\mathrm{p}=0.006)$ (Figure 3 ).

Comparison of the most common adverse events between Pfizer-BioNTech and Oxford-AstraZeneca vaccines was demonstrated in Table 5. It was observed that most of the side effects were significantly higher in OxfordAstraZeneca than Pfizer-BioNTech (P-value $<0.05$ ). Myalgia was the only adverse event that showed no significant difference between the two COVID-19 vaccines.

\section{Discussion}

Vaccines are considered the most effective intervention in public health, as they prevent and control the spread of 
Table 2 Post-Vaccination Symptoms of First and Second Doses of COVID-19 Vaccines

\begin{tabular}{|l|l|}
\hline Symptoms & Frequency (\%) \\
\hline Fever & $136(41.2)$ \\
Muscle and joint pain & $76(23)$ \\
Redness at the injection site & $4(1.2)$ \\
Myalgia & $121(36.71)$ \\
Malaise & $121(36.71)$ \\
Fatigue & $119(36.1)$ \\
Pain and swelling at the injection site & $65(19.7)$ \\
Chills & $46(13.9)$ \\
Headache & $86(24.2)$ \\
Diarrhea & $20(6.1)$ \\
Depression & $13(3.9)$ \\
Muscle tone & $16(4.9)$ \\
Dizziness & $27(8.2)$ \\
Cough & $11(3.3)$ \\
Nausea & $24(7.3)$ \\
Seizures & $3(0.9)$ \\
Anxiety & $7(2.1)$ \\
Dyspnea & $11(3.3)$ \\
Swollen lip and tongue & $3(0.9)$ \\
Sore throat & $24(7.3)$ \\
Anosmia & $4(1.2)$ \\
\hline
\end{tabular}

infectious diseases, therefore reducing mortality. However, as with other drugs, reactions may occur following vaccination. ${ }^{16}$ The majority of these reactions are neither severe nor frequent. Individuals' confidence in vaccines shows significant variation and relies on several factors, including awareness about vaccines, religious or political aspects, potential associated risks, and social and economic conditions. ${ }^{17}$ Moreover, it has been shown that people assess vaccination risks compared to other related risks differently than experts do. ${ }^{18}$

In this large-scale, community-based study in the Aseer region of the Kingdom of Saudi Arabia, we investigated adverse effects and infection rates following the administration of Pfizer-BioNTech and Oxford-AstraZeneca COVID-19 vaccines. Participants reported considerable systemic adverse effects, including fever, fatigue, headache, and muscle and joint pain, but very few reported having redness at the injection site, seizures, or other symptoms. Fever was observed to be the most common symptom. These findings are consistent with other recently published results, which reported that the most common post-injection side effects for first and second doses were fever, myalgia, fatigue, injection site pain, headache, joint pain, and chills. ${ }^{19-22}$

In the present study, vaccinated individuals reported adverse effects after both doses, with the majority occurring after the second dose. These findings are highly consistent with the published results of Phase III clinical trials of second doses of COVID-19 vaccines. . $^{6,9,23,24}$

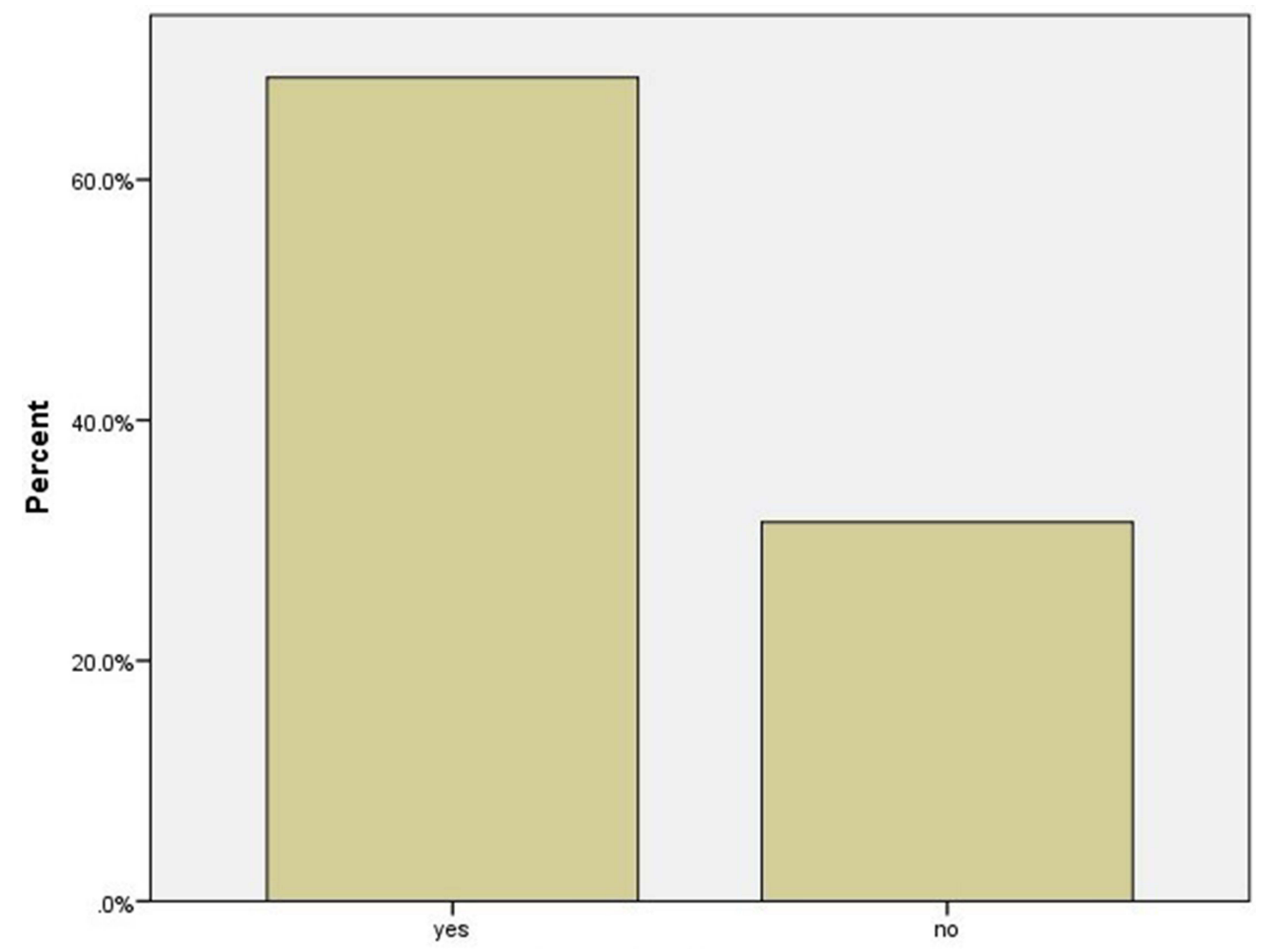

Figure I Frequency of post-vaccination symptoms. 
Table 3 The Onset of Symptoms in the First Dose and Second Dose of COVID-19 Vaccines

\begin{tabular}{|c|c|c|c|c|}
\hline Onset of Symptoms & First Dose Frequency (\%) & Second Dose Frequency (\%) & $\chi^{2}$ & P-value \\
\hline No symptoms after the first dose/second dose & $106(32.1)$ & $70(21.2)$ & \multirow{9}{*}{156.610} & \multirow{9}{*}{$<0.001$} \\
\hline$<5$ minutes & $10(3.0)$ & $14(4.2)$ & & \\
\hline $6-10$ minutes & $9(2.4)$ & $3(0.9)$ & & \\
\hline II-I5 minutes & $4(1.2)$ & $5(1.5)$ & & \\
\hline$>15$ minutes & $83(25.2)$ & $32(9.7)$ & & \\
\hline$<24$ hours & $85(25.8)$ & $\mathrm{I}(0.3)$ & & \\
\hline$<24$ hours & $5(1.5)$ & $204(6 \mid .8)$ & & \\
\hline$>24$ hours & $28(8.5)$ & $\mathrm{I}(0.3)$ & & \\
\hline Total & 330 & 330 & & \\
\hline
\end{tabular}

Although all COVID-19 vaccines cause similar side effects, it was found that participants who received the AstraZeneca vaccine reported significantly higher side effects than those who received the Pfizer-BioNTech vaccine. This is in line with results of previous studies, which stated that Pfizer-BioNTech was associated with lower side effects compared to Oxford-AstraZeneca and other vaccines. ${ }^{11,12}$ Consistently, the previous published results of phase III clinical trials and the fact sheets of the vaccines, which reported post-vaccination symptoms for those who received the second dose. ${ }^{6,7,9,23,24}$ Similar findings were also reported in a recent prospective observational study performed by Menni et al. ${ }^{8}$

The present study found the incidence of post-COVID-19vaccination side effects to be significantly more prevalent in males than in females. This finding contradicts that reported

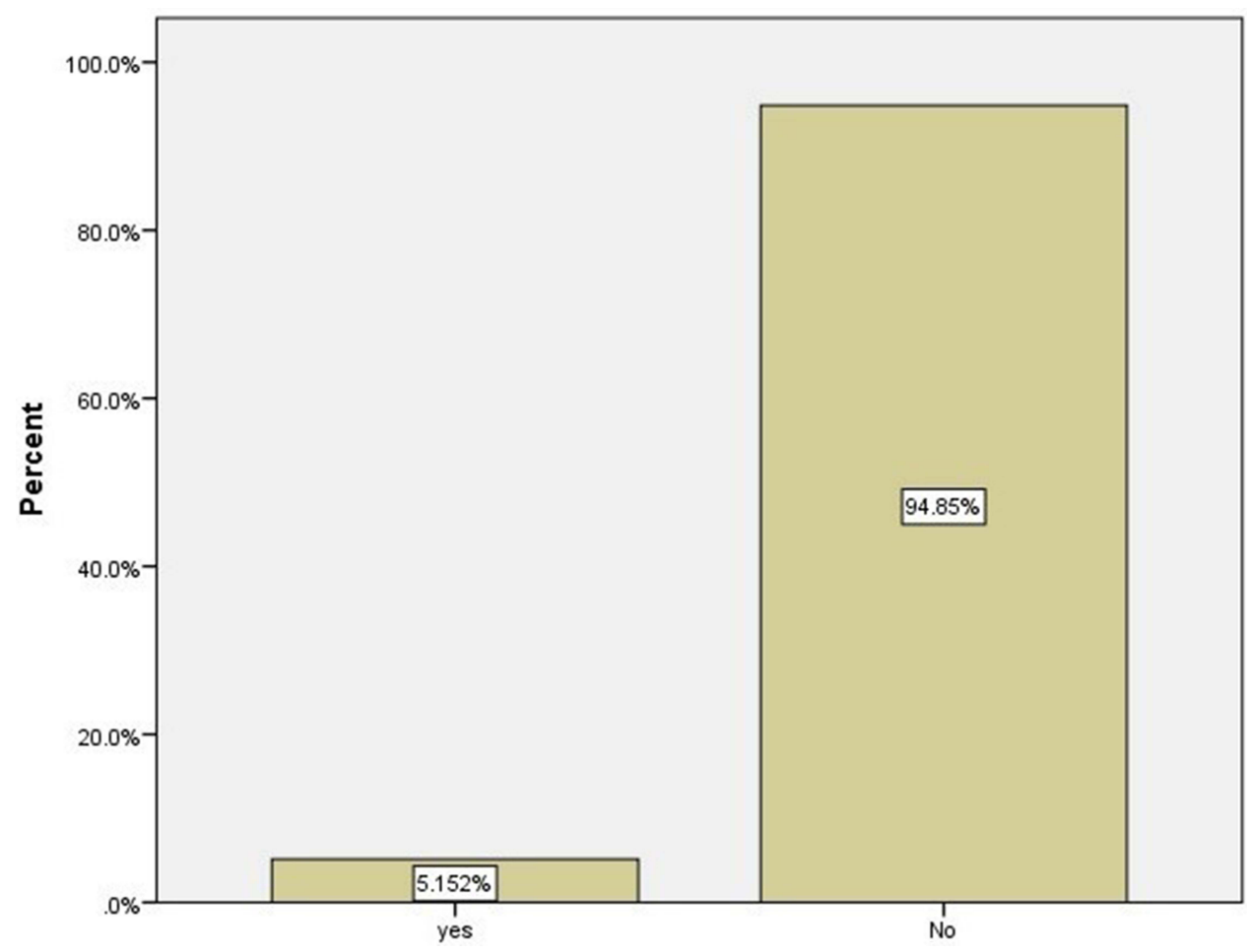

Figure 2 Percentage of participants who got infection with covid-19 after vaccination. 
Table 4 The Onset of Adverse Events of Side Effects in the Two Common Types of COVID-19 Vaccines

\begin{tabular}{|l|l|l|l|l|l|}
\hline \multirow{2}{*}{ Type of Vaccines } & \multicolumn{2}{l|}{ Post-Vaccination Symptoms } & \multirow{2}{*}{ Total } & \multirow{2}{*}{$\chi^{2}$} \\
\cline { 2 - 5 } & Yes & No & & \\
\cline { 1 - 5 } & $102(62.6 \%)$ & $61(37.4 \%)$ & $163(100.0 \%)$ & 5.209 & 0.022 \\
Oxizer/BioNTech & $124(74.3 \%)$ & $43(25.7 \%)$ & $167(100.0 \%)$ & \\
Total & $226(68.5 \%)$ & $104(31.5 \%)$ & $330(100.0 \%)$ & \\
\hline
\end{tabular}

by Alhazmi et al, ${ }^{25}$ who studied the short-term side effects of COVID-19 vaccines in Saudi people and found that the incidence was higher in females than males. The higher incidence of side effects in males in our study may be attributed to the lower proportion of females in our study sample.

The present study showed that many symptoms appeared within 15 minutes after injection of both vaccines. This finding is consistent with CDC guidelines, which recommend that all vaccine recipients be observed for a minimum of 15 minutes after injection; this is because it is mostly the inactive ingredients or excipients (including gelatine, egg protein, formaldehyde, neomycin, and thimerosal) that precipitate allergic reactions. $^{26}$
There are concerns about whether the vaccine can cause COVID-19; however, this is not possible, as these mRNA vaccines are not developed using the live SARS$\mathrm{CoV}-2$ virus. In the present study, approximately $5.15 \%$ of vaccinated participants still contracted the virus, this is because the body takes a few weeks after vaccination to produce T-lymphocytes and B-lymphocytes. Therefore, it is possible for a person to be infected with SARS-CoV-2 just before or just after vaccination and then get sick because the vaccine did not have enough time to provide protection. These cases are called breakthrough infections.

With increasing studies on the side effects of COVID-19 vaccines, public confidence in vaccine safety may improve, which would accelerate the vaccination process against

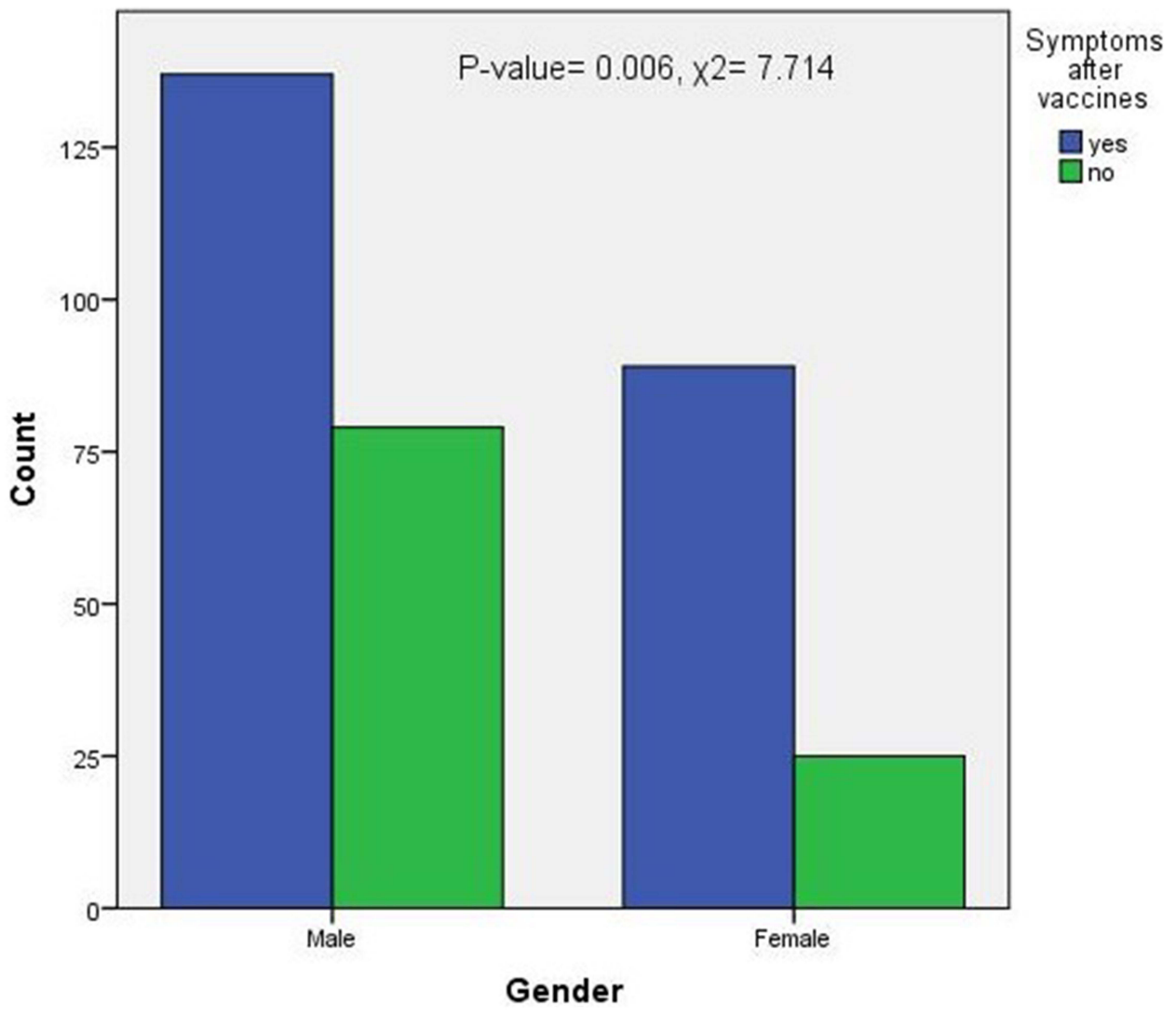

Figure 3 Comparison of post-vaccination side effects of first and second doses between males and females. 
Table 5 The Most Common Adverse Events of Side Effects for Pfizer and Oxford-AstraZeneca Vaccines

\begin{tabular}{|l|l|l|l|}
\hline Side Effects & Pfizer & Oxford-AstraZeneca & P-value \\
\hline Myalgia & $58(47.9 \%)$ & $63(52.1 \%)$ & 0.69 \\
Malaise & $47(38.8 \%)$ & $74(61.2 \%)$ & 0.004 \\
Fever & $46(33.8 \%)$ & $90(66.2 \%)$ & $>0.001$ \\
Fatigue & $46(38.7 \%)$ & $73(61.3 \%)$ & 0.003 \\
Muscle and joint pain & $27(35.5 \%)$ & $49(64.5 \%)$ & 0.006 \\
Headache & $21(26.3 \%)$ & $59(73.8 \%)$ & $<0.001$ \\
\hline
\end{tabular}

COVID-19. The findings of this study are of interest to health professionals and clinicians; the results raise awareness of COVID-19 vaccine safety, effectiveness, benefits, as no severe health problems were reported. These findings provide evidence against misunderstandings and conspiracy beliefs about the post-vaccination symptoms of COVID-19 vaccines, which increase vaccine hesitancy.

\section{Limitations}

This study faced some limitations. Some participants may not have fully reported all applicable data, resulting in a reporting bias. Moreover, community-based surveys were challenging to achieve during the COVID-19 pandemic. Additionally, the present study did not cover the whole country; it was only located in the southern Aseer region. Further studies are recommended to cover the entire Kingdom of Saudi Arabia to confirm the initial results of this study. The recommended studies of postvaccination side effects should distinguish between side effects that emerged after the first dose, second dose, and both doses.

\section{Conclusion}

The most common post-COVID-19-vaccination side effects reported by participants who received the Pfizer-BioNTech and Oxford-AstraZeneca vaccines were fever, myalgia, malaise, fatigue, muscle and joint pain, and headache, with very few having seizures, anosmia, or swollen lip and tongue. Adverse events were more common in participants who received the Oxford-AstraZeneca vaccine than in those who received the Pfizer-BioNTech vaccine. Further studies on vaccine safety are strongly recommended to strengthen public confidence in COVID-19 vaccines.

\section{Acknowledgments}

The authors acknowledge and appreciate the sponsorship of the Deanship of Scientific Research at King Khalid
University for funds provided through the General Research Program (grant number GRP/290/42).

\section{Funding}

Funds were provided by the Deanship of Scientific Research at King Khalid University through the General Research Program (grant number GRP/290/42).

\section{Disclosure}

The authors report no conflicts of interest for this work.

\section{References}

1. Kourlaba G, Kourkouni E, Maistreli S, et al. Willingness of Greek general population to get a COVID-19 vaccine. Glob Health Res Policy. 2021;6(1):1-10. doi:10.1186/s41256-021-00188-1

2. Sadarangani M, Marchant A, Kollmann TR. Immunological mechanisms of vaccine-induced protection against COVID-19 in humans. Nat Rev Immunol. 2021;21:475-484. doi:10.1038/s41577-021-00578-z

3. National Center for Immunization and Respiratory Diseases. Centers for Disease Control and Prevention. Interim clinical considerations for use of covid-19 vaccines are currently authorized in the United States. [updated May 6, 2021]. Available from: https://www.cdc.gov/vaccines/ covid-19/clinical-considerations/covid-19-vaccines-us.html. Accessed May 17, 2021

4. Algaissi AA, Alharbi NK, Hassanain M, Hashem AM. Preparedness and response to COVID-19 in Saudi Arabia: building on MERS experience. J Infect Public Health. 2020;13:834-838. doi:10.1016/j. jiph.2020.04.016

5. Saudi Arabia to make coronavirus vaccine free for citizens and residents. Arab News; 2020. Available from: https://arab.news/8u269. Accessed May 17, 2021.

6. Polack FP, Thomas SJ, Kitchin N, et al. Safety and efficacy of the BNT162b2 mRNA Covid-19 vaccine. $N$ Engl $J$ Med. 2020;383:2603-2615. doi:10.1056/NEJMoa2034577

7. Voysey M, Clemens SAC, Madhi SA, et al. safety and efficacy of the ChAdOx1 nCoV-19 vaccine (AZD1222) against SARS-CoV-2: an interim analysis of four randomized controlled trials in Brazil, South Africa, and the U.K. Lancet. 2021;397:99-111. doi:10.1016/S01406736(20)32661-1

8. Menni C, Klaser K, May A, et al. Vaccine side-effects and SARS-CoV-2 infection after vaccination in users of the COVID Symptom Study app in the U.K.: a prospective observational study. Lancet Infect Dis. 2021;21:939-945. doi:10.1016/S1473-3099(21)00224-3

9. El-Shitany NA, Harakeh S, Badr-Eldin SM, et al. Minor to moderate side effects of Pfizer-BioNTech COVID-19 vaccine among Saudi residents: a retrospective cross-sectional study. Int J Gen Med. 2021;14:1389-1401. doi:10.2147/IJGM.S310497 
10. Meo SA, Bukhari IA, Akram J, Meo AS, Klonoff DC. COVID-19 vaccines: comparison of biological, pharmacological characteristics and adverse effects of Pfizer/BioNTech and Moderna vaccines. Eur Rev Med Pharmacol Sci. 2021;25(3):1663-1669. doi:10.26355/ eurrev_202102_24877

11. Andrzejczak-Grządko S, Czudy Z, Donderska M. Side effects after COVID-19 vaccinations among residents of Poland. Eur Rev Med Pharmacol Sci. 2021;25(12):4418-4421. doi:10.26355/ eurrev_202106_26153

12. Beattie M, Murphy DJ, Atherton I, et al. Instruments to measure patient experience of healthcare quality in hospitals: a systematic review. Syst Rev. 2015;4:97. doi:10.1186/s13643-015-0089-0

13. Mathers N, Fox N, Hunn A. Surveys and questionnaires. The NIHR Research Design Service for Yorkshire \& the Humber. [updated March 11, 2009]. Available from: https://www.rds-yh.nihr.ac.uk/ wpcontent/uploads/2013/05/12_Surveys_and_Questionnaires_ Revision_2009.pdf. Accessed July 12, 2021.

14. Artino AR Jr, La Rochelle JS, Dezee KJ, et al. Developing questionnaires for educational research: AMEE Guide No. 87. Med Teach. 2014;36(6):463-474. doi:10.3109/0142159X.2014.889814

15. Ministry of Health - Kingdom of Saudi Arabia. MOH publications: COVID-19 guidelines. [updated July 7, 2021]. Available from: https://www.moh.gov.sa/en/Ministry/MediaCenter/Publications/ Pages/365 covid19.aspx. Accessed July 17, 2021

16. King Abdullah Petroleum Studies and Research Center. Saudi Arabia Coronavirus disease (COVID-19) situation. [updated April 17, 2021]. Available from: https://datasource.kapsarc.org/explore/dataset/saudiarabia-coronavirus-disease-covid-19-situation-demographics/. Accessed May 12, 2021.

17. Larson HJ, Cooper LZ, Eskola J, Katz SL, Ratzan S. Addressing the vaccine confidence gap. Lancet. 2011;378(9790):526-535. doi:10.1016/S0140-6736(11)60678-8

18. Gust DA, Woodruff R, Kennedy A, Brown C, Sheedy K, Hibbs B. Parental perceptions surrounding risks and benefits of immunization. Semin Pediatr Infect Dis. 2003;14:207-212. doi:10.1016/S10451870(03)00035-9
19. World health organization: Coronavirus disease (COVID-19): Vaccines safety. [updated February 17, 2021]. Available from: https://www.who.int/news-room/q-a-detail/coronavirus-disease(covid-19)-vaccines-safety. Accessed July 29, 2021.

20. Hatmal MM, Al-Hatamleh MA, Olaimat AN, et al. Side effects and perceptions following COVID-19 vaccination in Jordan: a Randomized, Cross-Sectional Study Implementing Machine Learning for Predicting Severity of Side Effects. Vaccines. 2021;9 (6):556. doi:10.3390/vaccines9060556

21. Riad A, Pokorná A, Attia S, Klugarová J, Koščík M, Kluger M. Prevalence of COVID-19 vaccine side effects among healthcare workers in the Czech Republic. J Clin Med. 2021;10(7):1428. doi:10.3390/jcm10071428

22. Kadali RAK, Janagama R, Peruru S, et al. Adverse effects of COVID-19 mRNA-1273 vaccine: a randomized, cross-sectional study on healthcare workers with detailed self-reported symptoms. J Med Virol. 2021;93(7):4420-4429. doi:10.1002/jmv.26996

23. Kadali RAK, Janagama R, Perry S, Malayala SV. Side effects of BNT162b2 mRNA COVID-19 vaccine: a randomized, cross-sectional study with detailed self-reported symptoms from healthcare workers. Int $J$ Infect Dis. 2021;106:376-381. doi:10.1016/j.ijid.2021.04.047

24. Chapin-Bardales J, Gee J, Myers T. Reactogenicity following receipt of mRNA-based COVID-19 vaccines. JAMA. 2021;325(21):2201. doi:10.1001/jama.2021.5374

25. Alhazmi A, Alamer E, Daws D, et al. Evaluation of side effects associated with COVID-19 vaccines in Saudi Arabia. Vaccines. 2021;9(6):674. doi:10.3390/vaccines9060674

26. Kesselheim AS, Darrow JJ, Kulldorff M, et al. An overview of vaccine development, approval, and regulation, with implications for COVID-19: analysis reviews the Food and Drug Administration's critical vaccine approval role with implications for COVID-19 vaccines. Health Aff. 2021;40(1):25-32. doi:10.1377/ hlthaff.2020.01620
Patient Preference and Adherence

\section{Publish your work in this journal}

Patient Preference and Adherence is an international, peer-reviewed, open access journal that focusing on the growing importance of patient preference and adherence throughout the therapeutic continuum. Patient satisfaction, acceptability, quality of life, compliance, persistence and their role in developing new therapeutic modalities and compounds to optimize clinical outcomes for existing disease

\section{Dovepress}

states are major areas of interest for the journal. This journal has been accepted for indexing on PubMed Central. The manuscript management system is completely online and includes a very quick and fair peer-review system, which is all easy to use. Visit http:// www.dovepress.com/testimonials.php to read real quotes from published authors. 